\title{
Design and Analysis of a Helium-Assisted Hybrid Drone for Flight Time Enhancement
}

\author{
Geronimo Macias ${ }^{\mathrm{a}}$, Kooktae Lee $^{\mathrm{a}}$ \\ ${ }^{a}$ Department of Mechanical Engineering, New Mexico Institute of Mining and Technology, 801 Leroy \\ Pl., Socorro, 87801, New Mexico, USA
}

\begin{abstract}
In this paper, a new design of a helium-assisted hybrid drone is proposed for the flight time enhancement. As is widely known, most of the drones with a VTOL (vertical take-off and landing) feature has a short operation time, limiting their capability to carry out sustainable operations for the given missions. Thus, with the clear goal of enhancing the flight time, this study aims to develop a hybrid drone system, where a helium balloon is used to provide a lifting force for this purpose. The proposed design for the hybrid drone has several benefits including easiness to manufacture and a relatively small size when compared to other types of hybrid drones. Various analyses are conducted for the design of the hybrid drone system including the balloon shape and size, buoyant force, flight time, and connector design.

Since the stability and performance are one of the most important issues for the new design, the pole location analysis is conducted based on the control theory. This rigorous analysis provides that the proposed hybrid drone design is stable as well as robust against swinging motions. To validate the effectiveness of the proposed design and flight time enhancement, simulations were conducted and experimental results are also provided using the manufactured hybrid drone system. Through the real experiments, it is proved that the hybrid drone can increase the flight time more than 2.5 times while guaranteeing stable motions.
\end{abstract}

Keywords: Hybrid Drone, Helium UAV, Drone Design, Flight Time Increase, UAV Stability and Performance Analysis

\section{Introduction}

Advances in drone technologies have enabled various types of drones (alternatively UAVs) to be used in numerous applications and missions including wildlife and environment monitoring [1, 2, 3, 4], cellular network $[5,6,7]$, military $[8,9]$, planetary exploration $[10,11,12]$, entertainment $[13,14,15]$, smart farming $[16,17]$, search and surveillance $[18,19]$, to list a few. Unlike fixed wing UAVs, drones with a VTOL (vertical take-off and landing) feature have wide applicability since they can hover over a particular point or region and do not require runways. As a trade-off, this type of drones (like quadcopters) can only fly in a relatively small amount of time (e.g., usually up to 20 mins [20, 21]), which unfortunately limits their usability for long endurance flights. For instance, in comparison between a fixed-wing aircraft and a mulitrotor for environmental mapping, it is shown that mutltirotor UAVs yield more accurate results due to its hovering capability while its flight endurance is a limiting factor [22].

To increase the flight time of VTOL drones, there have been many approaches including fixed wing drones combined with VTOL capability [23], hybrid drones supported by extra lifting force [24, 25], use of a solar-power energy harvesting method [20], use of a laser power beam from a ground station to wirelessly transfer power to a UAV in motion [26], an in-flight battery switching method that requires a flying battery to dock on the main UAV to provide an alternative power source [27], and many others. However, the previous approaches have several limitations such as their sizes and/or difficulty in manufacturing due to their unique designs. In what follows, we provide more detailed literature surveys on some related works to better understand what researches have been done for this purpose, followed by the contribution of this research. 
Literature Survey: The "S-CLOUD" UAV designed by Hwan Song et al. [24, 25] and shown in Fig. 1 (a) posed a minimal risk to people near its flying radius because of its torus-shaped envelope. This envelope is manufactured from thin polyethylene terephthalate (PET) that surrounds the coaxial rotors and 2-axis crossed flaps, which control the S-CLOUD drone. The torus-shaped envelope is filled with helium to provided a lifting force such that the flight time of the drone is increased. The uniqueness of the design is that the on-board electronics such as the flight controller, LIDAR sensors, RC receiver, electronic speed controller, LiPo battery, servo motors, and coaxial brushless DC motors and rotors are located at the center of the torus-shaped envelope. The total weight of the S-CLOUD is $550 \mathrm{~g}$ and the lifting force provided by the helium balloon is $490 \mathrm{~g}$, which leaves $60 \mathrm{~g}$ to be lifted by the thrust from the rotors. This UAV has a flight time of approximately 63 minutes, which is more than that of standard VTOL drones such as quadcopters. The downside of the design is that the torus-shaped enveloped is difficult to fabricate due to its hollow shape and the aerial dynamics of the UAV are complicated.

Wan et al. [28] presented a $200.3 \mathrm{~g}$ solar-powered blimp for the purpose of increasing the flight time efficiency of the UAV. Blimps are a type of lighter-than-air UAVs which rely on their neutral buoyancy to stay afloat as shown in Fig. 1 (b). Some of the advantages of blimps are their low power consumption, ease of take-off and landing, and their capability for long endurance flights. The downside of using a blimp is that it can be considerably larger in size when compared to a standard UAV, which makes blimps an undesirable option for accessing locations such as caves. Furthermore, the blimp has a limited payload capacity and is difficult to control due to its inherent sensitiveness to disturbances such as wind and temperature.

Cho et al. [21] developed the Georgia Tech Miniature Autonomous Blimp (GT-MAB) that has a long flight duration of up to two hours per battery charge. Like the S-CLOUD UAV, the GT-MAB UAV is safe for human-robot interactions. The GT-MAB UAV weighs $85.9 \mathrm{~g}$ and has a low payload capacity of 12.1 $\mathrm{g}$, which is a limiting factor in the design. Because of the low payload capacity, the researchers designed a small and lightweight driving and sensing hardware systems to control the blimp. The researchers utilized a proportional-integral-derivative (PID) controller to realize autonomy and achieve stable flights. The benefits of blimps over other UAVs, including a cheap cost, reduced power consumption, and an increase in safety, have been noted in several research papers.

Gonzalez et al. [29] used a $200 \mathrm{~g}$ modified Plantraco RC blimp with the goal of developing a lowcost autonomous indoor blimp. Because of its indoor application, the blimp and its components were designed such that the size of the blimp and the weight of the on-board hardware were minimized. The authors implemented a PID and fuzzy logic controllers to aid the blimp in navigating through two different environments. One of the drawbacks of the blimp is its low payload capacity because it relies on its neutral buoyancy to stay afloat. Its low payload capacity restricted the choice of sensors that were used in the design.

Lonneville et al. [30] used helium balloons for aerial photography. The benefit of using helium balloons is that there are no electronic components and the system can stay afloat until most of the helium permeates through the material. The downside of using just a helium balloon is that the balloon can easily be influenced by external stimuli such as wind, therefore, this simple design would be undesirable because there is no way to stabilize the helium balloon due to its lacking of control mechanisms.

A blade-free drone (Fig. 1 (c)) that utilizes several microblowers to generate a propulsive force that maneuvers the drone in any direction in the three-dimensional space was developed in [31]. The drawback of this blade-free drone is that it cannot perform pitching and rolling types of rotation because the center of gravity, by design, is placed at the bottom of the airframe.

Contribution: In this work, we aim to develop a helium-assisted hybrid drone for the flight time enhancement while overcoming the major drawbacks of previous system designs. The contributions of our works can be summarized as follows:

1) The proposed design for the hybrid drone system is simple enough with a low manufacturing process and cost. One of the benefits for the proposed design is that any off-the-shelf VTOL drones and helium balloons can be directly purchased from markets to build the hybrid drone without manufacturing them from scratch. What we propose in this research is the whole system design with the core component connecting the balloon with the drone. 


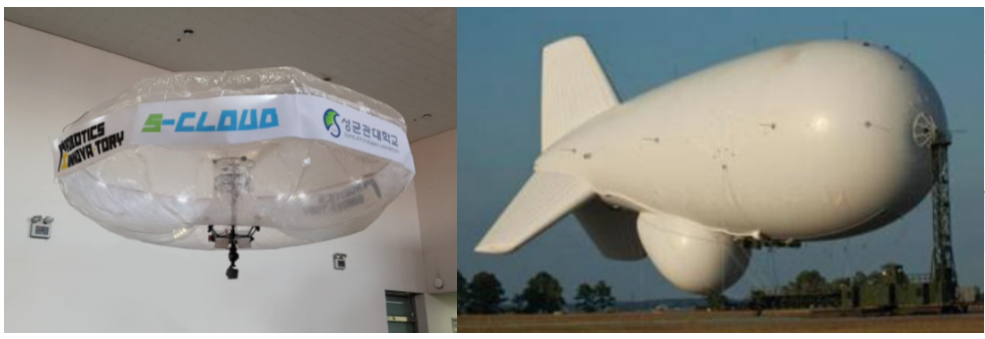

(a) S-CLOUD

(b) Blimp UAV

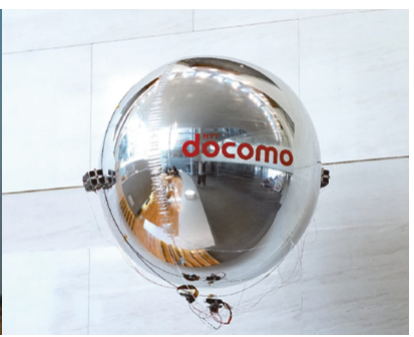

(c) Fanless drone

Figure 1: Various types of helium-assisted drones

2) Analyses on the balloon shape and size as well as the lifting force are provided. These results suggest an appropriate shape and size of the balloon while providing a corresponding lifting force for the system.

3) The size of the hybrid drone system can be relatively small when compared to existing ones. In some applications (e.g, cave explorations using a drone for the mapping purpose), the size is a limiting factor and it cannot be too large.

4) According to the provided calculus, the flight time is guaranteed to be a certain amount. At the same time, this calculus can be utilized to suggest a required volume of the helium balloon to achieve a preset value of the flight time.

5) Stability and performance are analyzed using the control theory (system pole location analysis). Two different hybrid drone designs with a fixed-angle connector (rigid attachment) and a ball-and-socket joint (non-rigid attachment) are compared based on the control theory, suggesting which design is better in terms of the stability and robustness against oscillations.

The rest of the paper is organized as follows. A brief explanation about the hybrid drone and conceptual design are introduced, followed by several problems to solve in Section 2. Details about the design of the helium-assisted hybrid drone are presented in Section 3. Section 4 delivers the stability and performance analysis of the hybrid drone system with two different design. To validate the proposed concept, various results from both simulations and experiments are provided in Section 5. Finally, Section 6 concludes the paper.

\section{Problem Description}

For sustainable operations of drone systems, it is necessary to increase the flight time. Most of the conventional drones having hovering capability such as quadcopters and hexacopters can fly around 20 mins, which may not be enough for many missions requiring a long-time operation.

The major problem we want to tackle in this study is thus to design a hybrid drone system, which is easy to manufacture, small enough, and easy to control while maintaining a long flight time. The conceptual drawing of the helium-assisted hybrid drone system is illustrated in Fig. 2.

In the proposed concept, any VTOL drones can be used as a drone platform and the helium balloon will be attached to a top support frame. This top support frame will be then connected to the quadcopter using some connecting part such as a ball-and-socket joint. The benefit of the proposed hybrid drone system is that it is unnecessary to manufacture both actuators and balloon since they can be both purchased from markets, which greatly simplifies the manufacturing process. The only parts that need to be designed and manufactured are the top support frame and a connecting part, which can be printed using a 3D printer.

Although the proposed design yields a simple manufacturing process, it does not guarantee the stable motion and easiness of control for the hybrid drone system. There are several important issues need to be considered for the design and control of the hybrid system as follows: 1) Shape of the balloon; 2) Size of the balloon to provide an enough lifting force for sustainable operations; 3) Design of a connecting part; and 4) 


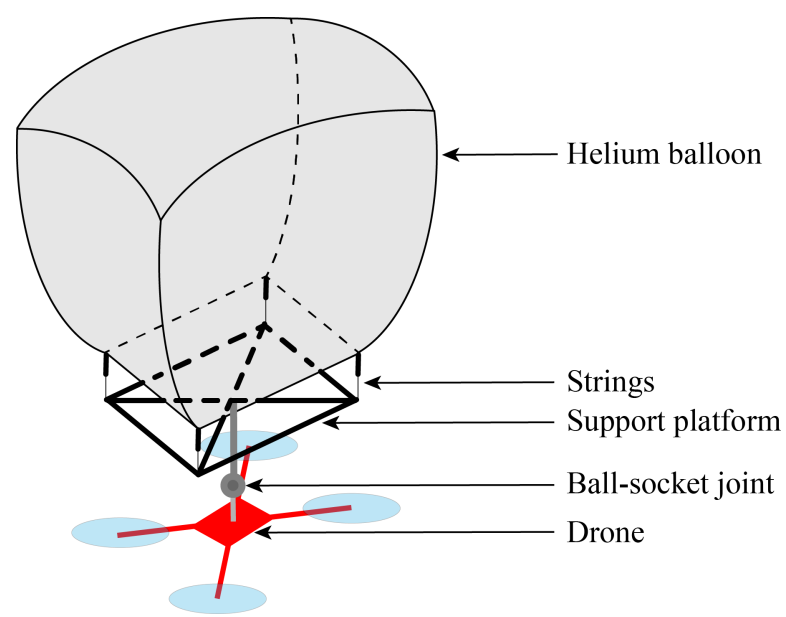

Figure 2: Shows the components of the hybrid drone.

Stability and performance analysis. In this paper, we will investigate above issues for the proposed hybrid drone system design. Each problem will be addressed in detail, followed by the solution to resolve them in separate sections.

\section{Design of the Hybrid Drone}

\subsection{Analysis on the Helium Balloon Shape}

Among various shapes of the helium balloon such as cuboid or sphere as a candidate for the hybrid drone system, two major factors need to be considered: a size constraint and a lifting force. Although the size constraint depends on the application where the hybrid drone system will be applied, we set this size constraint as $0.75 \mathrm{~m} \times 0.75 \mathrm{~m} \times 0.55 \mathrm{~m}$ for the width, length, and height of the balloon, targeting cave exploration missions where the average passage size of caves is known to be $0.75 \mathrm{~m} \times 0.75 \mathrm{~m}$ for the width and height according to [32]. Due to the trade-off between the size constraint and lifting force, analysis on the shape is performed. This analysis will lead to which shape is better in terms of the larger lifting force while conforming with the given size constraint.

In Fig. 3 (a), numerical analysis is provided to determine the shape of the balloon with the lifting force analysis between the two different options: cuboid and sphere. The x-axis is for the area of the balloon (the mid-plane area in the sphere case) and double y-axes are for the ratio of the characteristics height, which is defined as the height (held constant) for the cuboid and as the diameter for the sphere, to lifting force (left) and the volume itself (right). For comparison between the cuboid and sphere, the lifting force to the characteristic height was determined for various volumes of the cuboid and sphere balloons. The lifting force can be determined from the volume calculation based on the fact that one liter of helium can lift one gram of mass.

In the analysis, we constrained the mid-plane diameter of the sphere to be the same as the length and width of the cuboid balloon as illustrated in Fig. 3 (b). We defined the area term to be the product of length and width of the cuboid balloon. The area term was set initially to be $0.1 \mathrm{~m}^{2}$, therefore, the length, width, and diameter were determined by taking the square root of the area term. Thereafter, the area term was increased by an increment of $0.0025 \mathrm{~m}^{2}$. We also constrained the height of the cuboid balloon to be $0.55 \mathrm{~m}$ for all cases. For the sphere, we did not impose any constraints, therefore, the mid-plane diameter changed due to the change in the cuboid area term. The volumes of both shapes were determined for comparison purposes.

From Fig. 3 (a), we can observe that the cuboid balloon has a larger volume than the sphere balloon for the range of values in the x-axis. From the figure, we can also observe that the lifting force to the characteristic height ratio is greater for the cuboid balloon, indicating that the cuboid balloon will provide 


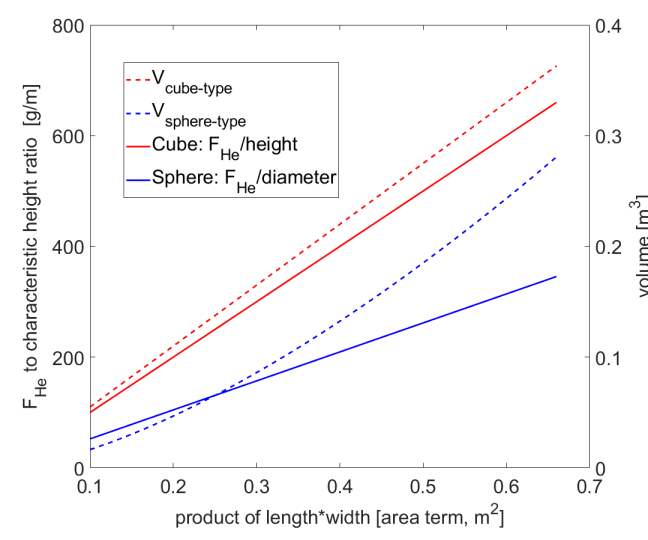

(a)

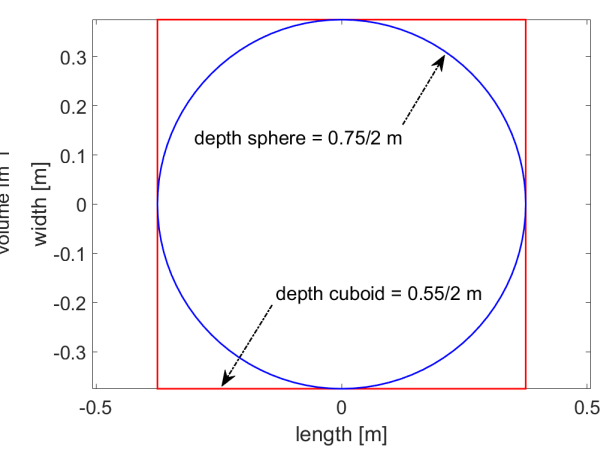

(b)

Figure 3: (a) The left and right y-axis show the the lifting force to characteristic height ratio and the volume of the sphere and cuboid balloon, respectively, plotted against the area term in the x-axis; and (b) schematic of the mid-plane cross sections for the cuboid and spherical balloons.

more lifting force. Based on this analysis, one can conclude that the cuboid balloon is better than the sphere for our design because of its larger volume and lifting force to the characteristic height ratio.

\subsection{Lifting Force and Flight Time Analysis}

\subsubsection{Lifting Force Analysis}

The first prototype of the hybrid drone was constructed by using the nine off-the-shelf balloons as shown in Fig. 4. In this way, one can easily construct the hybrid drone system without manufacturing the balloon. Although this first prototype achieved several successful test flights, it turns out that the entire size of the balloons becomes bigger than expected due to the large density of the balloon material requiring more balloons for enough lifting force as well as the unnecessary spaces between balloons. However, this may be a good option if there is no size constraint on the hybrid drone system since the balloons can be directly purchased from markets.

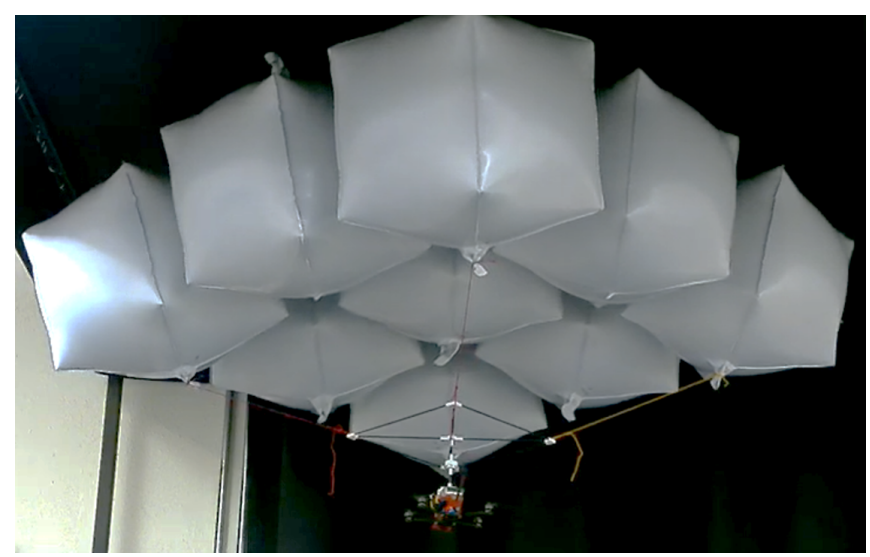

Figure 4: First prototype of the hybrid drone system using the nine off-the-shelf cubic-type balloons purchased from markets.

To reduce the size of the whole system, we manufactured the cuboid balloon. The material used to manufacture the balloon is the 1 mil poly sheet which has a density of $960.56 \mathrm{~kg} / \mathrm{m}^{3}$. 


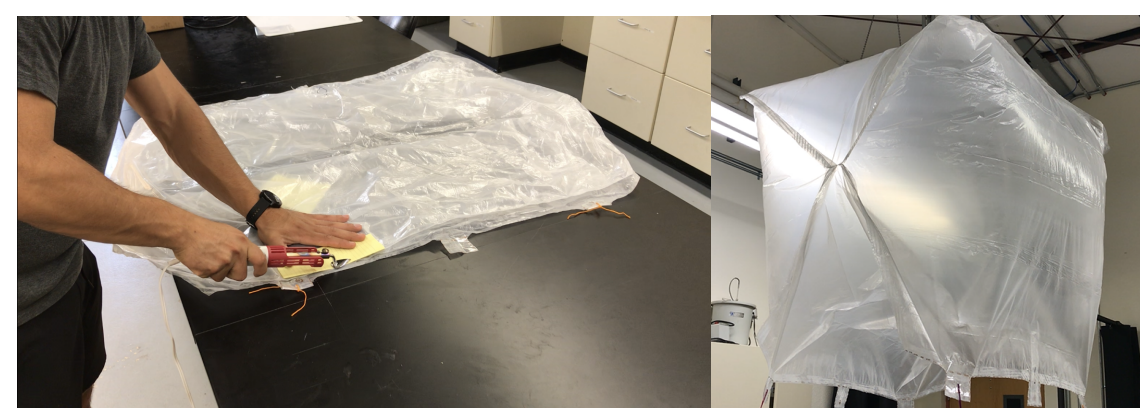

(a)

(b)

Figure 5: (a) Picture for the manufacturing process, where a small iron is used to bond the 1 mil poly sheet faces of the balloon; and (b) Manufactured balloon with dimensions of $0.75 \mathrm{~m} \times 0.75 \mathrm{~m} \times 0.55 \mathrm{~m}$. The weight of the balloon is $82.8 \mathrm{~g}$.

The dimension of the manufactured balloon shown in Fig. 5 is set to be length $(L)=0.75 \mathrm{~m}$, width $(W)=0.75 \mathrm{~m}$, and height $(H)=0.55 \mathrm{~m}$, which corresponds to a volume of $0.31 \mathrm{~m}^{3}$. With the thickness of the 1 mil poly sheet $\left(2.54 \times 10^{-5} \mathrm{~m}\right)$ together with the given dimension of the manufactured balloon, the theoretical mass of the balloon is calculated by $67.7 \mathrm{~g}$. However, the actual weight was $15.1 \mathrm{~g}$ heavier than the theoretical value due to the excess material left on the perimeter of the balloon. In comparison to the first prototype, the cuboid balloon manufactured is 92.7 grams lighter. Another advantage of the manufactured balloon is that its lifting force is concentrated on the single manufactured balloon rather than nine.

The total weight of the hybrid drone system including the drone, battery, support platform, ball-andsocket joint, and the balloon before filling it with the helium was $430.7 \mathrm{~g}$. Depending on the volume of the helium filled in the balloon, the total weight of the hybrid drone system changes since it provides a lifting force. To maximize the lifting force by helium, the balloon needs to be fully filled with the helium, which, however, may damage the balloon in the worst case, recalling that it is made from a thin poly sheet material. Thus, it is indispensable to analyze how much the helium gas can fill the balloon with a corresponding lifting force. To this end, the plot is provided in Fig. 6 to present the total weight of the hybrid drone system and lifting force with a variation of the helium percentage fill. Based on this plot, we determined that the actual balloon is filled to $94 \%$ of its capacity, resulting in the lifting force of $345.4 \mathrm{~g}$ with the weight of helium itself $47.7 \mathrm{~g}$. Therefore, the total weight of the hybrid drone system with $94 \%$ filled by helium is approximately computed by $430.7-345.4+47.7=133 \mathrm{~g}$.

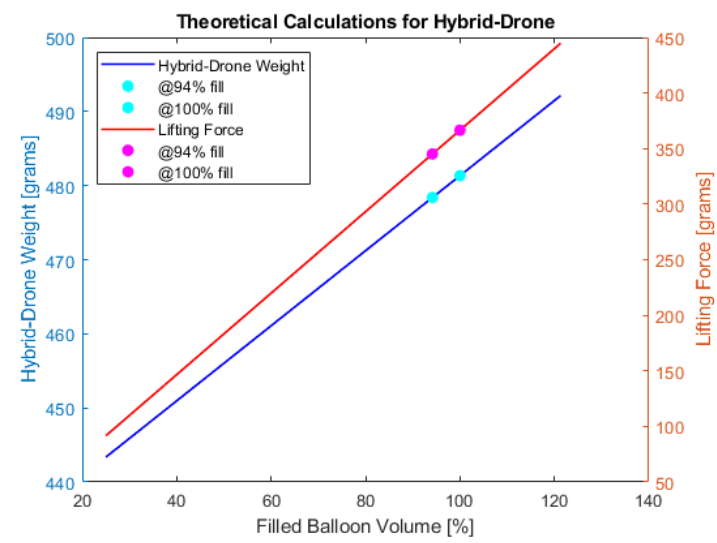

Figure 6: Theoretical analysis on the hybrid drone weight and the lifting force with a variation of the percentage helium volume in the given balloon size. 


\subsubsection{Flight Time Increase Analysis}

By harnessing the lifting force from the helium balloon, the hybrid drone can fly longer than the original one. To analyze how much the flight time can increase using the helium balloon, the following flight time calculus is provided:

$$
T=\frac{C \times M}{i_{\text {avg }}} \times 60=\frac{C \times M \times V}{m_{\text {total }} \times P} \times 60[\text { mins }],
$$

where the meaning of all variables are presented in Table 1 and the second equality in the above equation holds by $i_{\text {avg }}=\frac{m_{\text {total }} \times P}{V}$.

\begin{tabular}{|l|l|}
\hline Variable & Description \\
\hline \hline$m_{\text {total }}$ & Total mass of the hybrid drone system \\
$i_{\text {avg }}$ & Average current draw by the drone \\
$P$ & Power to weight ratio (how efficient the motors are at lifting \\
& one unit of weight) \\
$V$ & Battery pack voltage \\
$C$ & Battery capacity in mAh \\
$M$ & Battery discharge margin (e.g., 80\% of its capacity to pro- \\
& tect the battery from permanent damage) \\
\hline
\end{tabular}

Table 1: Variables for the flight time calculus

It is worth noting that in (1), all system parameters except for the total mass $m_{\text {total }}$ are constant for a given quadcopter even if a helium balloon is attached because they are all quadcopter-dependent parameters. The only variable affected by the helium balloon is $m_{\text {total }}$. According to (1), it is known that the flight time is directly inverse proportional to $m_{\text {total }}$, which can be reduced by the hellium balloon. For instance, the total flight time of the hybrid drone will be doubled when $m_{\text {total }}$ becomes a half of the original quadcopter system.

For our quadcopter used in experiments, the value of each parameter is given as follows: $m_{\text {total }}=347.9$ $\mathrm{g}, i_{\text {avg }}=3.4 \mathrm{~A}, P=144.64 \mathrm{~W}, V=14.8 \mathrm{~V}, C=1300 \mathrm{mAh}$, and $M=0.8$, resulting in the total flight time $T_{\text {quadcopter }}=18.35$ mins. The helium balloon provides a total of weight reduction by 214.9 $\mathrm{g}(=347.9 \mathrm{~g}-133 \mathrm{~g})$, which increases the total flight time based on the above analysis by $T_{h y b r i d}=$ $\frac{1300 \mathrm{mAh} \times 0.8 \times 14.8 \mathrm{~V}}{133 \mathrm{~g} \times 144.64 \mathrm{~W}} \times 60=48 \mathrm{mins}$, which is about 2.62 times greater than the original quadcopter flight time.

\subsection{Design Consideration: Fixed-Angle Connector vs. Ball-and-Socket Joint}

To attach the helium balloon to the quadcopter, a support platform and joint were manufactured. The support platform is made of $3 \mathrm{~mm}$ carbon fiber rods and 3D printed joints to hold the frame together. The dimension of the support platform is $0.279 \mathrm{~m} \times 0.279 \mathrm{~m}$.

For the connector and joint design, we considered two different options: a 3D printed fixed-angle connector (FAC) and a ball-and-socket joint (BSJ) for the attachment of the drone to the support platform. The FAC, as shown in Fig. 7, is more compact and it allows for the simultaneous movement between the drone and the balloon, i.e., if the drone rolls to the left, then the balloon will also move to the left. However, the movement will require a large thrust to tilt the hybrid drone in the direction of travel due to the resistance of rotation from the helium balloon.

On the other hand, the BSJ, as shown in Fig. 8, enables the hybrid drone to have some tilting freedom when a roll or pitch action is being applied. The tilting freedom is due to the ball rotating freely (with minimal friction) inside the socket. The balloon is also decoupled from the yawing motion of the drone, which allows the drone to rotate freely about the $x-y$ plane.

More stringent analysis for the control of two different options will be provided in the following section based on the control theory. 


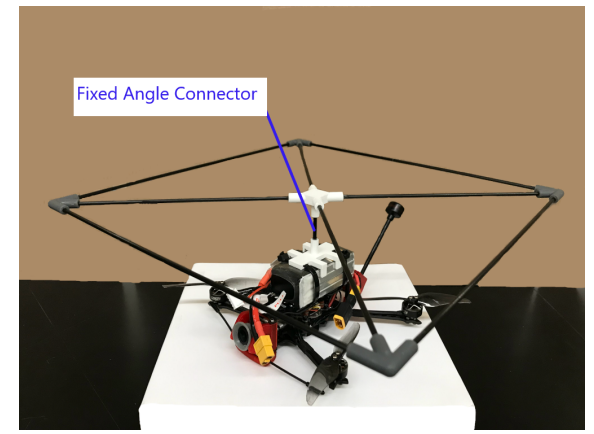

(a)

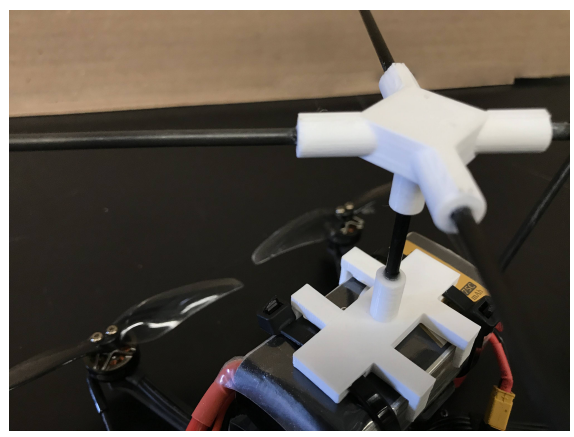

(b)

Figure 7: Hybrid drone with the FAC attachment: (a) the overall view and (b) close-up view of the FAC attachment.

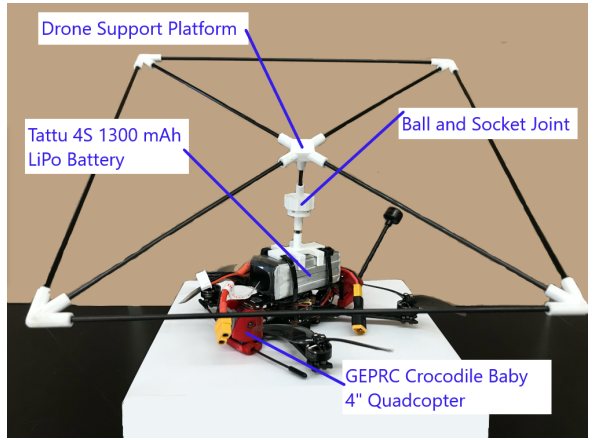

(a)

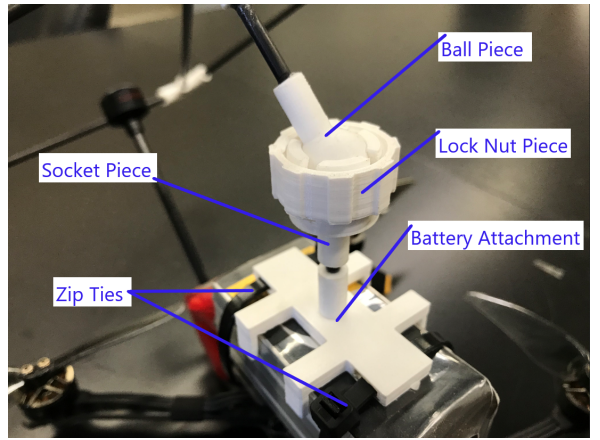

(b)

Figure 8: Hybrid drone with the BSJ attachment: (a) the overall view and (b) close up view of the BSJ attachment.

\section{Stability and Performance Analysis of Hybrid Drone System}

This section provides stability and performance (especially convergence speed) analysis for the planar motion of the hybrid drone system based on the control theory. The planar motion schematic for the two distinct designs are presented in Fig. 9 (a) and (b), respectively. In both cases, the angle $\phi$ is given to denote the tilt motion (i.e., roll or pitch) of the quadcopter with respect to the equilibrium angle, i.e. $\phi=0^{\circ}$. The notation of variables is provided in Table 2 .

In the case of FAC, the helium balloon and the quadcopter rotate together due to the fixed-angle connector as illustrated in Fig. 9 (a). When the thrusts $F_{1}$ and $F_{2}$ are adjusted to stabilize the quadcopter, which will lift the hybrid drone in the upward direction, the airflow around the balloon will be formed as shown in blue solid lines in Fig. 9 (a). The largest drag force will then take place at point Q. Based on this aerodynamics effect analysis, the rotational equation of planar motion in the FAC case is obtained as follows:

- Equation of planar motion for the FAC case:

$$
I_{x x}^{F A C} \ddot{\phi}=\left(F_{1}-F_{2}\right) l+h_{o} F_{H e} \sin \phi+F_{Q}\left(l_{b} \cos \phi-\left(h_{b}+h_{o}\right) \sin \phi\right),
$$

where the meaning of each variable is shown in Fig. 9 (a) and $F_{Q}$ is the exerting force on the point $Q$ by the drag. Linearizing the above equation with a small angle assumption leads to

$$
I_{x x}^{F A C} \ddot{\phi}=\left(F_{1}-F_{2}\right) l+\left(F_{H e} h_{o}-F_{Q}\left(h_{b}+h_{o}\right)\right) \phi+F_{Q} l_{b}
$$




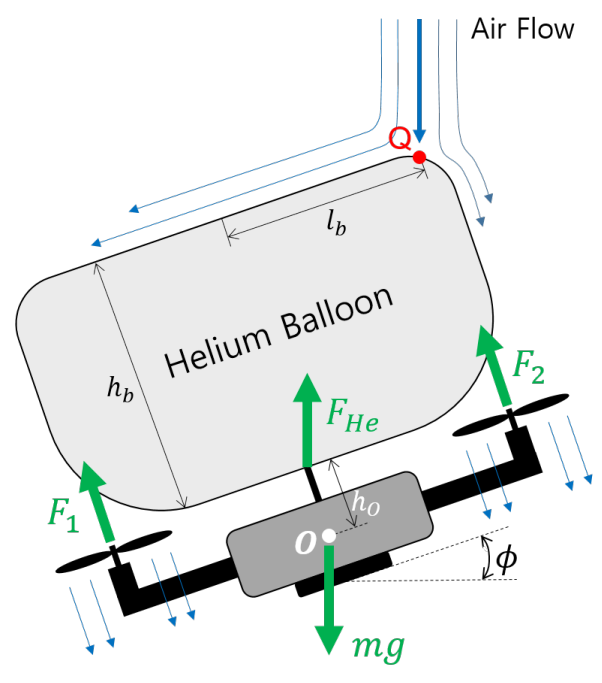

(a) FAC

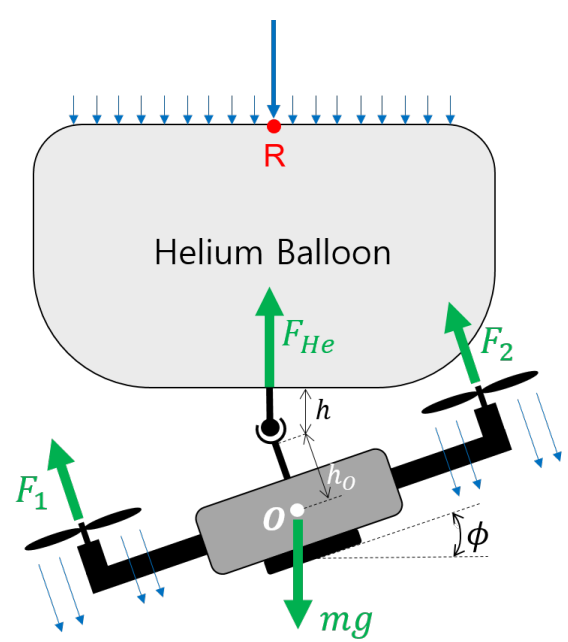

(b) BSJ

Figure 9: Planar motion schematic of the hybrid drone with (a) fixed-angle connector (FAC); and (b) ball-and-socket joint (BSJ)

\begin{tabular}{|l|l|}
\hline Variable & Description \\
\hline \hline$m$ & Mass of the quadcopter \\
$g$ & Gravity \\
$l$ & Planar motion angle \\
$h_{o}$ & Length of the quadcopter arm in the planar motion \\
& Distance from the quadcopter mass center $O$ to the point \\
$h$ & shown in Fig. 9 \\
$h_{b}$ & Length of the top rod in the ball-and-socket joint \\
$l_{b}$ & Height of the balloon \\
$F_{1}$ & Half width of balloon \\
$F_{2}$ & Thrust by motor 1 \\
$F_{H e}$ & Lifting force by the Helium balloon \\
$F_{Q}$ & Drag force acting on the point $Q$ in the FAC case \\
$F_{R}$ & Drag force acting on the point $R$ in the BSJ case \\
$I_{x x}^{F A C}$ & Moment of inertia in the FAC case \\
$I_{x x}^{B S J}$ & Moment of inertia in the BSJ case \\
\hline
\end{tabular}

Table 2: Variables of the hybrid drone system for FAC and BSJ cases

Given the definition of state vector $x=[\phi, \dot{\phi}]^{T}$ and control input $u=\left[F_{1}, F_{2}\right]^{T}$, the state-space representation is written by

$$
\dot{x}=A^{F A C} x+B^{F A C} u+G
$$

where $A^{F A C}=\left[\begin{array}{cc}0 & 1 \\ \frac{\left(F_{H e}-F_{Q}\right) h_{o}-F_{Q} h_{b}}{I_{x x}^{F A C}} & 0\end{array}\right], B^{F A C}=\left[\begin{array}{cc}0 & 0 \\ \frac{l}{I_{x x}^{F A C}} & \frac{-l}{I_{x x}^{F A C}}\end{array}\right]$, and $G=\left[\begin{array}{c}0 \\ F_{Q} l_{b}\end{array}\right]$.

Then, applying the Laplace transform gives us

$$
s X(s)-x(0)=A^{F A C} X(s)+B^{F A C} U(s)+G
$$


With a P-type state feedback control $U(s)=-K X(s)$, the above equation becomes

$$
\begin{gathered}
s X(s)-x(0)=\left(A^{F A C}-B^{F A C} K\right) X(s)+G \\
\Rightarrow X(s)=\left(s I-A_{K}^{F A C}\right)^{-1}(x(0)+G),
\end{gathered}
$$

where $A_{K}^{F A C}=A^{F A C}-B^{F A C} K$.

On the other hand, in case of BSJ, the equation of planar motion is obtained as follows.

- Equation of planar motion for the BSJ case:

$$
I_{x x}^{B S J} \ddot{\phi}=\left(F_{1}-F_{2}\right) l+\left(F_{H e}-F_{R}\right) h_{o} \sin \phi
$$

Similarly, linearization with a small angle assumption results in

$$
I_{x x}^{B S J} \ddot{\phi}=\left(F_{1}-F_{2}\right) l+\left(F_{H e}-F_{R}\right) h_{o} \phi
$$

Given the definition of state vector $x=[\phi, \dot{\phi}]^{T}$ and control input $u=\left[F_{1}, F_{2}\right]^{T}$, the state-space representation is written by

$$
\dot{x}=A^{B S J} x+B^{B S J} u
$$

where $A^{B S J}=\left[\begin{array}{cc}0 & 1 \\ \frac{\left(F_{H e}-F_{R}\right) h_{o}}{I_{x x}^{B S J}} & 0\end{array}\right]$, and $B^{B S J}=\left[\begin{array}{cc}0 & 0 \\ \frac{l}{I_{x x}^{B S J}} & \frac{-l}{I_{x x}^{B S J}}\end{array}\right]$.

Applying the Laplace transform With a state feedback control $U(s)=-K X(s)$ yields

$$
\begin{gathered}
s X(s)-x(0)=\left(A^{B S J}-B^{B S J} K\right) X(s) \\
\Rightarrow X(s)=\left(s I-A_{K}^{B S J}\right)^{-1} x(0),
\end{gathered}
$$

where $A_{K}^{B S J}=A^{B S J}-B^{B S J} K$.

Notice that there are two major factors that result in a considerable difference between the two options for the stability as well as the performance of the hybrid drone. The first factor is " $G$ " in (3), which, however, does not appear in (5), since it affects the movement of the drone as illustrated in Fig. 9 (a). The second factor, which is the most significant source for the performance of the hybrid drone, is the polar moment of inertia $I_{x x}$. As the movement of the balloon is strictly tied together with the quadcopter in the FAC case while they are separated in the BSJ case, $I_{x x}^{F A C}$ is much greater than $I_{x x}^{B S J}$. The convergence speed of angle $\phi$ to the stable point (zero degree) is then analyzed by the eigenvalue of $A_{K}^{F A C}$ and $A_{K}^{B S J}$ as follows.

With the state feedback controller gain $K=\left[\begin{array}{ll}k_{11} & k_{12} \\ k_{21} & k_{22}\end{array}\right]$, we have

$$
\begin{aligned}
A_{K}^{F A C} & =A^{F A C}-B^{F A C} K=\left[\begin{array}{cc}
0 & 1 \\
\frac{\left(F_{H e}-F_{Q}\right) h_{o}-F_{Q} h_{b}}{I_{x x}^{F A C}} & 0
\end{array}\right]-\left[\begin{array}{cc}
0 & 0 \\
\frac{l}{I_{x x}^{F A C}} & \frac{-l}{I_{x x}^{F A C}}
\end{array}\right]\left[\begin{array}{ll}
k_{11} & k_{12} \\
k_{21} & k_{22}
\end{array}\right] \\
& =\left[\begin{array}{cc}
0 & 1 \\
c^{F A C} & d^{F A C}
\end{array}\right],
\end{aligned}
$$

where $c^{F A C}=\frac{\left(F_{H e}-F_{Q}\right) h_{o}-F_{Q} h_{b}-\left(k_{11}-k_{21}\right) l}{I_{x x}^{F A C}}$ and $d^{F A C}=-\frac{\left(k_{12}-k_{22}\right) l}{I_{x x}^{F A C}}$

and

$$
\begin{aligned}
A_{K}^{B S J} & =A^{B S J}-B^{B S J} K=\left[\begin{array}{cc}
0 & 1 \\
\frac{\left(F_{H e}-F_{R}\right) h_{o}}{I_{x x}^{B S J}} & 0
\end{array}\right]-\left[\begin{array}{cc}
0 & 0 \\
\frac{l}{I_{x x}^{B S J}} & \frac{-l}{I_{x x}^{B S J}}
\end{array}\right]\left[\begin{array}{ll}
k_{11} & k_{12} \\
k_{21} & k_{22}
\end{array}\right] \\
& =\left[\begin{array}{cc}
0 & 1 \\
c^{B S J} & d^{B S J}
\end{array}\right]
\end{aligned}
$$


where $c^{B S J}=\frac{\left(F_{H e}-F_{R}\right) h_{o}-\left(k_{11}-k_{21}\right) l}{I_{x x}^{B S J}}$ and $d^{B S J}=-\frac{\left(k_{12}-k_{22}\right) l}{I_{x x}^{B S J}}$.

Then, the eigenvalue of two different cases are calculated from $\operatorname{det}\left(\lambda^{(\cdot)} I-A_{K}^{(\cdot)}\right)=0$ by

$$
\begin{aligned}
& \lambda^{(\cdot)}\left(\lambda^{(\cdot)}-d^{(\cdot)}\right)-c^{(\cdot)}=0 \\
& \Rightarrow \lambda^{(\cdot)}=\frac{d^{(\cdot)} \pm \sqrt{\left(d^{(\cdot)}\right)^{2}+4 c^{(\cdot)}}}{2}
\end{aligned}
$$

With an appropriate choice of $K$ such that $d^{(\cdot)}<0$ and $\left(d^{(\cdot)}\right)^{2}+4 c^{(\cdot)}<0$, one can always guarantee the hybrid drone is stable for both FAC and BSJ. However, their performances are significantly different due to the fact that $I_{x x}^{F A C} \gg I_{x x}^{B S J}$. In this case, the convergence speed strictly depends on the magnitude of $d^{(\cdot)}$ (the square root term becomes purely imaginary from the above assumption), resulting in $\left|d^{F A C}\right| \ll\left|d^{B S J}\right|$. Thus, the angle $\phi$ for the case of BSJ will converge to the zero degree much faster than that for the case of FAC. We provide both simulation and experimental results in Section 5 for the validation of analysis.

\section{Simulations and Flight Time Experiments}

Based on the pole location analysis in Section 4, stability and performance are analyzed for the two different design options. These results provide justification on which design is preferable in terms of the stability and performance. The effects of the connector length in both designs on the hybrid drone system are also analyzed. These simulation results can be used to determine more preferable design of the hybrid drone system. Real flight tests are carried out for both design options, which validates the analysis.

\subsection{Simulations for Stability and Performance Analysis}

For the stability and performance analysis, we carried out various simulations while changing a parameter to check its effect on the system. Particularly, the major consideration is the comparison between the two different design options: FAC and BSJ.

The parameters used in the simulation are provided in Table 3.

\begin{tabular}{|l|l|l|}
\hline Variable & Description & Value \\
\hline \hline$g$ & Gravity & $9.81 \mathrm{~m} / \mathrm{s}^{2}$ \\
$l$ & Length of quadcopter arm in the planar motion & $0.1 \mathrm{~m}$ \\
$h_{o}$ & Distance from the quadcopter mass center $O$ to & $0.05 \mathrm{~m}-0.15 \mathrm{~m}$ \\
& the bottom side of balloon (FAC) / to the ball- & \\
$h_{q}$ & and-socket joint (BSJ) & \\
$h_{b}$ & Height of the rectangle quadcopter & $0.015 \mathrm{~m}$ \\
$l_{b}$ & Height of the balloon & $0.55 \mathrm{~m}$ \\
$F_{H e}$ & Half width of balloon & $0.375 \mathrm{~m}$ \\
$F_{Q}$ & Lifting force by the Helium balloon & $0.2 \mathrm{~g}$ \\
$F_{R}$ & Drag force in the FAC case & $10 \%$ of $F_{H e}$ \\
$k_{11}$ & Drag force in the BSJ case & $10 \%$ of $F_{H e}$ \\
$k_{12}$ & Controller gain & 3.5 \\
$k_{21}$ & Controller gain & 0.1 \\
$k_{22}$ & Controller gain & 0.01 \\
$\phi(0)$ & Controller gain & 0.01 \\
$\dot{\phi}(0)$ & Initial angle & $3^{\circ}$ \\
\hline
\end{tabular}

Table 3: Variables and their values used in the simulation for the performance analysis

The difference between the two design options mainly stems from the polar moment of inertia, which directly affects the pole locations. Although the real hybrid drone planar geometry is complicated as shown 
in Fig. 10 (a), a simplified model in Fig. 10 (b) is considered for the approximated calculation of the polar moment of inertia. Based on this simplified model, the polar moment of inertia is calculated and provided in Appendix A.

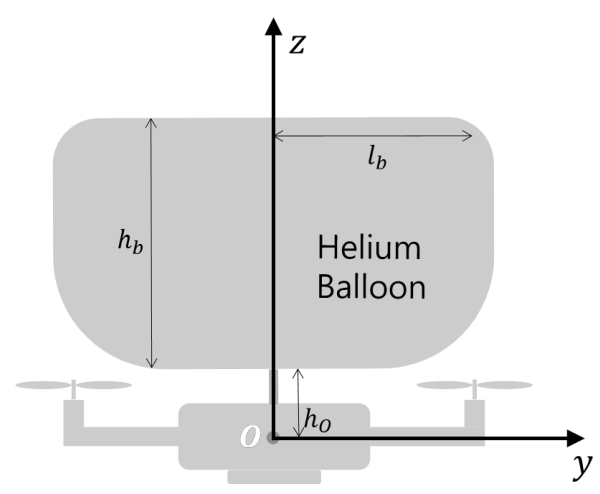

(a) Planar geometry of the hybrid drone

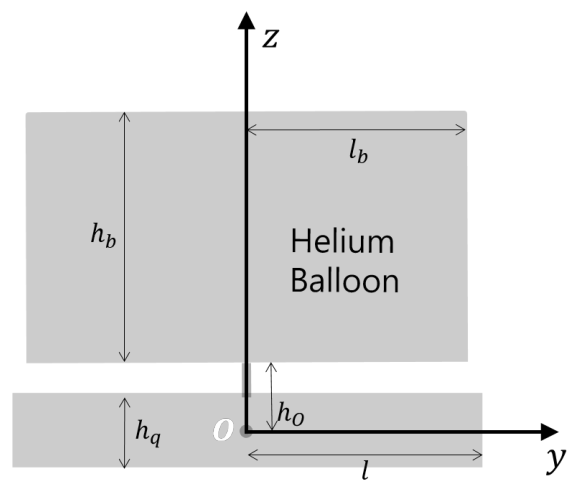

(b) Simplified planar geometry

Figure 10: Planar geometry of (a) hybrid drone and (b) simplified one

The polar moment of inertia can be divided by two parts: the helium balloon and the quadcopter, both of which are approximated by rectangles with given dimensions in Fig. 10 (b). Given the assumption that the density of two rectangles $\left(\rho_{\text {balloon }}\right.$ and $\left.\rho_{\text {quad }}\right)$ with a unit depth $(1 \mathrm{~m})$ is uniform over the area in the rectangle, the polar moment of inertia for the FAC and BSJ case is, respectively, obtained by $I_{x x}^{F A C}=0.0107$ $\mathrm{m}^{4}$ and $I_{x x}^{B S J}=0.0018 \mathrm{~m}^{4}$. (See Appendix A for details.)

From the above values, it turns out that $I_{x x}^{F A C}$ is almost 6 times greater than $I_{x x}^{B S J}$. This difference results in the pole locations as indicated by cross mark symbols in Fig. 11. In this root locus-type plot, it is

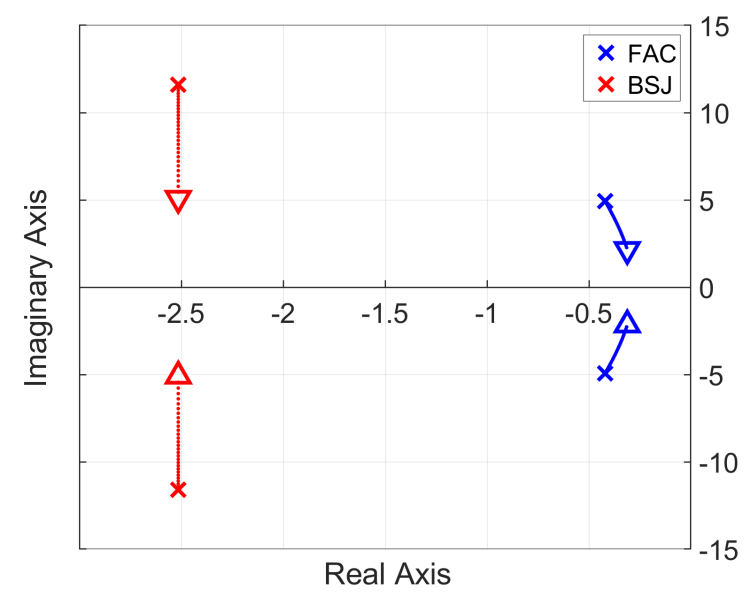

Figure 11: Variation of pole locations for the planar motion of the hybrid drone system with two different joints (FAC and BSJ): the symbols "x", triangles, and dots indicate when $h_{o}=0.05 m, h_{o}=0.15 m$, and between these two-end values by linearly increasing $h_{o}$, respectively

observed that both FAC and BSJ designs are stable as the real part of poles are placed in the left-half plane. However, the speed of convergence to the equilibrium point (i.e., $\phi=0^{\circ}$ ) when the angle $\phi$ of the hybrid drone is nonzero is quite different because of the magnitude of the real part of poles. The convergence speed 
for the BSJ case will be almost 6 times faster than that for the FAC case as we have $\operatorname{Real}\left(\lambda^{F A C}\right)=-0.4216$ and $\operatorname{Real}\left(\lambda^{B S J}\right)=-2.5148$.

Another factor needs to be analyzed is the length of connector $h_{o}$ since the pole locations also vary depending on the value of $h_{o}$. For this purpose, the value of $h_{o}$ is given as a variable linearly spacing from $0.05 \mathrm{~m}$ to $0.15 \mathrm{~m}$. The resultant pole locations are also presented in Fig. 11 with dot symbols. The triangle symbols are given to indicate the end-value of $h_{o}$ (i.e., $h_{o}=0.15 \mathrm{~m}$ ). From this result, we notice that increasing $h_{o}$ yields less frequency in oscillation of $\phi$ for both FAC and BSJ cases. In the FAC case, the convergence speed of $\phi$ will decrease as well, which is not desirable, because the magnitude of the real part in the poles decreases.

To better visualize the output response of $\phi$ in time, we provided two different plots for the output response to initial conditions in Figs. 12. Fig. 12 (a) and (b) correspond to the case when $h_{o}=0.05 \mathrm{~m}$ and

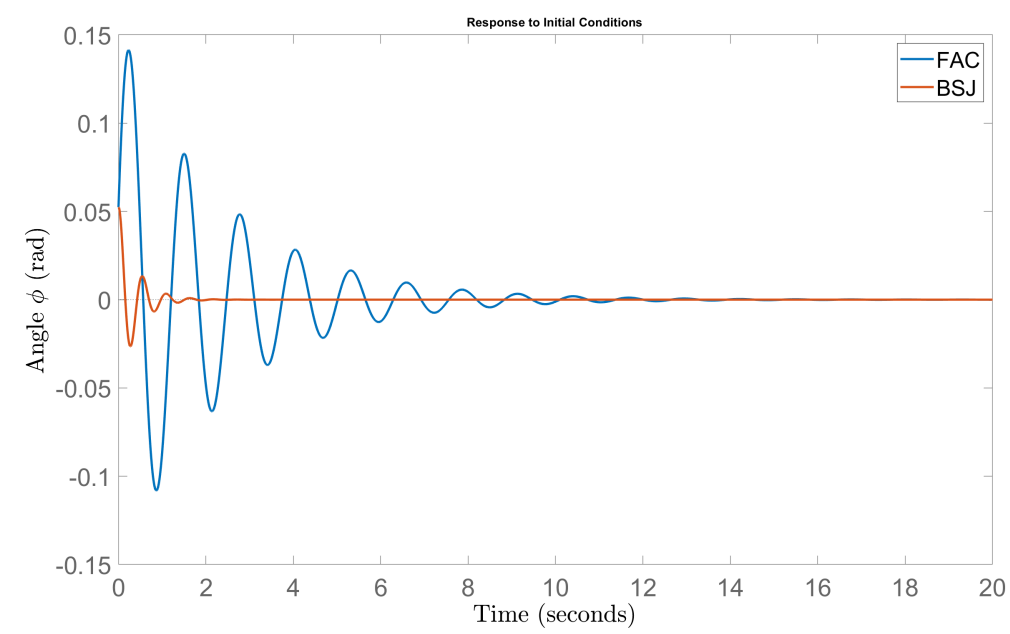

(a)

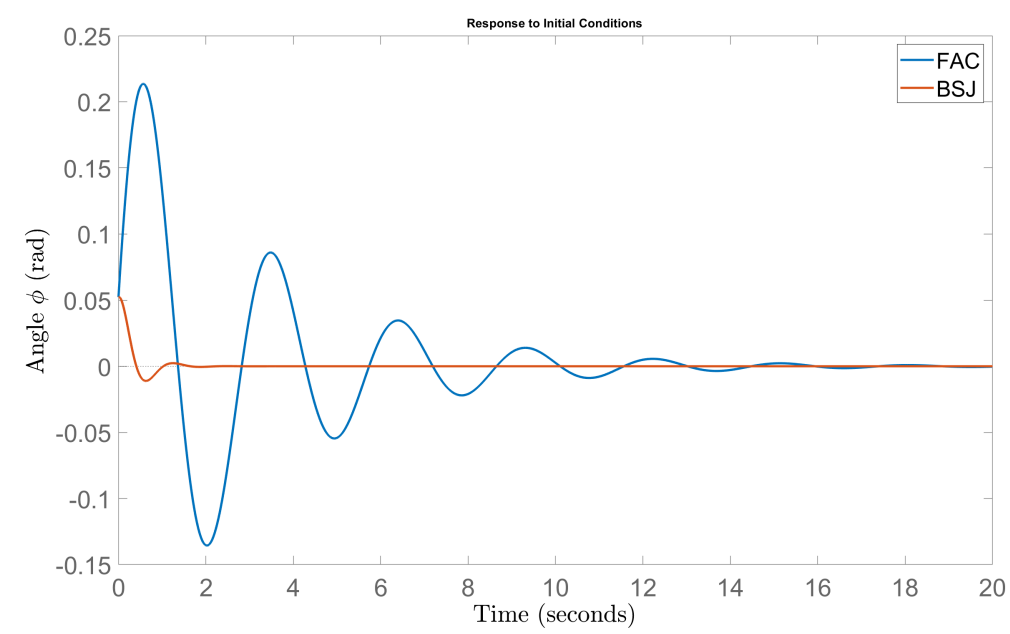

(b)

Figure 12: Time response plot for the angle $\phi$ with (a) $h_{o}=0.05 \mathrm{~m}$ and (b) $h_{o}=0.15 \mathrm{~m}$

$h_{o}=0.15 \mathrm{~m}$, respectively, where initial conditions are given as $\phi=3^{\circ}$ and $\dot{\phi}=1^{\circ} / \mathrm{s}$ as described in Table 3. In both cases (a) and (b), it is observed that the BSJ design provides much faster convergence speed 
(less than 2 seconds) to the equilibrium point, whereas the angle for the FAC design very slowly converges to the zero angle (more than 12 seconds). Thus, the BSJ design is better than the FAC design. We provide experimental validations later for the real flight tests of FAC and BSJ designs as well, which coincides with the simulation analysis.

One more difference between Fig. 12 (a) and (b) is the oscillation (swing) frequency. For the lengthier $h_{o}$ design in Fig. 12 (b), the frequency of oscillation has decreased in both designs. While the magnitude of $\phi$ for the FAC also increases for larger $h_{o}$, this is not observed in the BSJ case. Thus, it is desirable to design a hybrid drone with the BSJ design having a lenghty connector $h_{o}$ for less swinging motions. Notice that $h_{o}$ cannot be greater than a certain value as it will cause an instability issue from the pole location analysis in Fig. 11. If one keeps increasing $h_{o}$, then two poles in the BSJ case will be separated on the real axis making one pole go to the left and the other to the right, which will cross the imaginary axis.

\subsection{Flight Test Experiments}

The GEPRC Crocodile Baby 4" quadcopter was used as the UAV of choice to conduct the experiments because of its light-weight frame design, compactness, and high efficiency power system. The components that came with the drone include GEPRC 1404 2750KV motor, Gemfan 4024 propellers, GEP-20A-F4 AIO flight control system, and GPS module (not used in our experiment). For the hybrid-drone presented in this work, a four-cell, $1300 \mathrm{mAh}$ Tattu Li-PO battery with a nominal output voltage of $14.8 \mathrm{~V}$ was selected, weighing 155 grams. The total weight of the drone (including battery) is approximately $315 \mathrm{~g}$. With the support platform the weight of the hybrid-drone increases to $347.9 \mathrm{~g}$. The video links are provided for the demonstration and embedded in the document.

FAC Attachment: The hybrid drone with the FAC attachment was more difficult to control ${ }^{1}$ than the BSJ attachment because the balloon is tightly coupled with the quadcopter, influencing the pitch, roll, and yaw angles of the drone. The hybrid drone is required to overcome the moment of inertia of the balloon. Figs. 13 (a) - (d) show that the hybrid drone with the FAC kept swinging, making this type more difficult to control than the BSJ-type hybrid drone as analyzed in simulations. The hybrid drone with the FAC attachment requires high control inputs to dampen swinging motions, which could be detrimental to extending the flight time of the hybrid drone because the motors will deplete the energy from the battery faster.

BSJ Attachment: Unlike the FAC case, the hybrid drone with the BSJ attachment was smooth to control $^{2}$ and did not present any noticeable instability issues when the pitch and roll of the drone were changed. Furthermore, the hybrid drone was pushed to its limit by increasing the control inputs rapidly for the test of robustness to oscillations but it was able to be stabilized relatively quickly. Figs. 13 (e) (h) show the snapshots of the BSJ-type hybrid drone for the flight tests. The hybrid drone with the BSJ attachment can rotate freely because of the BSJ attachment, and therefore can easily be positioned in the desired direction as if it had no balloon.

\footnotetext{
${ }^{1}$ https://youtu.be/u9rvZ1i01lc

${ }^{2}$ https://youtu.be/wmBjU23sMds
} 


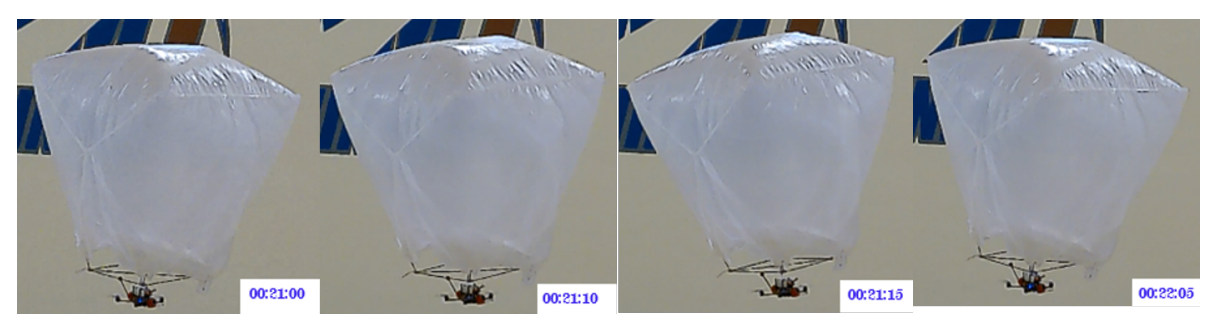

(a)

(b)

(c)

(d)

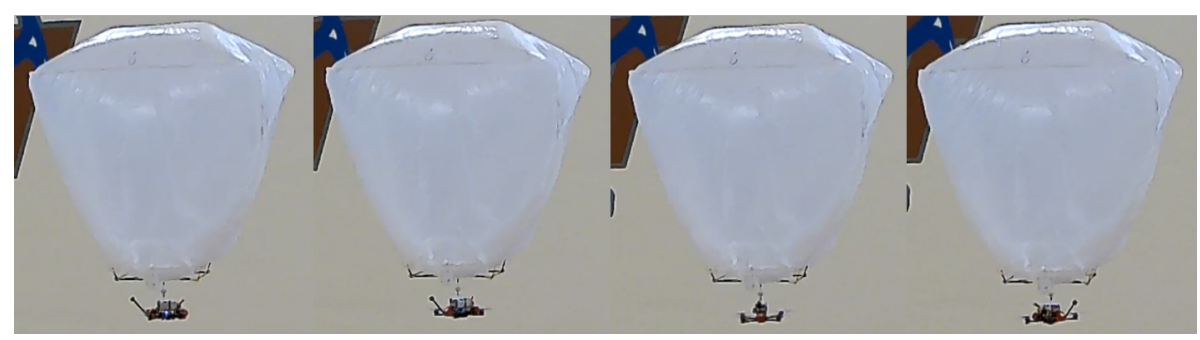

(e)

(f)

(g)

(h)

Figure 13: Sequence of flight test snapshots for the FAC case (a) - (d) and the BSJ case (e)-(h)

With the BSJ attachment, the hybrid drone flew more than 46 minutes ${ }^{3}$, whereas the original quadcopter without the balloon can fly up to 18 minutes, resulting in the increase of the total flight time more than 2.56 times when compared to its original flight time.

\section{Conclusion}

This paper proposed a new hybrid drone system design using helium gas to enhance the flight time. Unlike the existing ones, the proposed system is easy to manufacture and control while its size can be relatively small. For the hybrid drone system, the shape of the balloon was analyzed together with the lifting force analysis. To calculate the flight time enhancement, the flight time calculus is provided, theoretically guaranteeing that the hybrid drone system can fly more than 2.6 times longer than the original system.

Two different designs (FAC and BSJ) are considered to connect the balloon and the drone. For the stability and performance analysis of the two designs, the pole location analysis was carried out using the classical control theory. This rigorous analysis guarantees that the BSJ makes the system more stable and robust against swinging motions as the movements of drone can be decoupled from that of the balloon. Both simulation and experimental results were provided to validate the effectiveness of the proposed design and the flight time test. It was shown that the proposed hybrid drone system can increase the flight time more than 2.56 times with stable motions.

\footnotetext{
${ }^{3}$ https://youtu.be/4bgJWj4JrYg
} 


\section{Appendix A. Polar Moment of Inertia Calculus}

$$
\begin{aligned}
I_{x x}^{F A C}= & \iint r^{2} \rho(y, z) d A=\iint\left(y^{2}+z^{2}\right) \rho(y, z) d y d z \\
= & \int_{-h_{q} / 2}^{h_{q} / 2} \int_{-l}^{l}\left(y^{2}+z^{2}\right) d y d z \cdot \rho_{\text {quad }}+\int_{h_{o}}^{h_{o}+h_{b}} \int_{-l_{b}}^{l_{b}}\left(y^{2}+z^{2}\right) d y d z \cdot \rho_{\text {balloon }} \\
= & \left.\left(\frac{1}{3}\left(l^{3}+l^{3}\right)\left(\frac{h_{q}}{2}+\frac{h_{q}}{2}\right)+\frac{1}{3}(l+l)\left(\frac{h_{q}^{3}}{8}+\frac{h_{q}^{3}}{8}\right)\right)\right) \cdot \rho_{\text {quad }}+ \\
& \left(\frac{1}{3}\left(l_{b}^{3}+l_{b}^{3}\right)\left(\left(h_{o}+h_{b}\right)-\left(h_{o}\right)\right)+\frac{1}{3}\left(l_{b}+l_{b}\right)\left(\left(h_{o}+h_{b}\right)^{3}-h_{o}^{3}\right)\right) \cdot \rho_{\text {balloon }} \\
= & \left(\frac{2}{3} l^{3} h_{q}+\frac{1}{6} l h_{q}^{3}\right) \cdot \rho_{\text {quad }}+\left(\frac{2}{3} l_{b}^{3} h_{b}+\frac{2}{3} l_{b}\left(\left(h_{o}+h_{b}\right)^{3}-h_{o}^{3}\right)\right) \cdot \rho_{\text {balloon }} \\
= & 0.0018+0.0089=0.0107 \mathrm{~m}^{4}
\end{aligned}
$$

In the case of BSJ, however, the rotation of the balloon is decoupled from the quadcopter, leading to the following polar moment of inertia.

$$
\begin{aligned}
I_{x x}^{B S J} & =\int_{-h_{q} / 2}^{h_{q} / 2} \int_{-l}^{l}\left(y^{2}+z^{2}\right) d y d z \cdot \rho_{\text {quad }}=\left(\frac{2}{3} l^{3} h_{q}+\frac{1}{6} l h_{q}^{3}\right) \cdot \rho_{q u a d} \\
& =0.0018 \mathrm{~m}^{4}
\end{aligned}
$$

\section{Acknowledgement}

This research is supported by National Cave and Karst Research Institute (NCKRI) Internal Seed Grant.

\section{References}

[1] R. H. Kabir, K. Lee, Wildlife monitoring using a multi-uav system with optimal transport theory, Applied Sciences 11 (9) (2021) 4070.

[2] J. C. Hodgson, S. M. Baylis, R. Mott, A. Herrod, R. H. Clarke, Precision wildlife monitoring using unmanned aerial vehicles, Scientific reports 6 (1) (2016) $1-7$.

[3] G. M. Bolla, M. Casagrande, A. Comazzetto, R. Dal Moro, M. Destro, E. Fantin, G. Colombatti, A. Aboudan, E. C. Lorenzini, Aria: Air pollutants monitoring using uavs, in: 2018 5th IEEE International Workshop on Metrology for AeroSpace (MetroAeroSpace), IEEE, 2018, pp. 225-229.

[4] Z. Duan, Y. Li, J. Wang, G. Zhao, S. Svanberg, Aquatic environment monitoring using a drone-based fluorosensor, Applied Physics B $125(6)(2019) 1-8$.

[5] M. Mozaffari, A. T. Z. Kasgari, W. Saad, M. Bennis, M. Debbah, Beyond 5g with uavs: Foundations of a 3d wireless cellular network, IEEE Transactions on Wireless Communications 18 (1) (2018) 357-372.

[6] Y. Zeng, J. Lyu, R. Zhang, Cellular-connected uav: Potential, challenges, and promising technologies, IEEE Wireless Communications 26 (1) (2018) 120-127.

[7] M. Mozaffari, W. Saad, M. Bennis, Y.-H. Nam, M. Debbah, A tutorial on uavs for wireless networks: Applications, challenges, and open problems, IEEE communications surveys \& tutorials 21 (3) (2019) 2334-2360.

[8] T. Samad, J. S. Bay, D. Godbole, Network-centric systems for military operations in urban terrain: The role of uavs, Proceedings of the IEEE 95 (1) (2007) 92-107.

[9] M. A. Ma'Sum, M. K. Arrofi, G. Jati, F. Arifin, M. N. Kurniawan, P. Mursanto, W. Jatmiko, Simulation of intelligent unmanned aerial vehicle (uav) for military surveillance, in: 2013 international conference on advanced computer science and information systems (ICACSIS), IEEE, 2013, pp. 161-166.

[10] M. Bryson, S. Sukkarieh, Observability analysis and active control for airborne slam, IEEE Transactions on Aerospace and Electronic Systems 44 (1) (2008) 261-280.

[11] A. Elfes, S. S. Bueno, M. Bergerman, E. C. De Paiva, J. G. Ramos, J. R. Azinheira, Robotic airships for exploration of planetary bodies with an atmosphere: Autonomy challenges, Autonomous Robots 14 (2) (2003) 147-164.

[12] J. Balaram, M. Aung, M. P. Golombek, The ingenuity helicopter on the perseverance rover, Space Science Reviews 217 (4) (2021) 1-11. 
[13] D. Brescianini, M. Hehn, R. D'Andrea, Quadrocopter pole acrobatics, in: 2013 IEEE/RSJ International Conference on Intelligent Robots and Systems, IEEE, 2013, pp. 3472-3479.

[14] M. Hehn, R. D'Andrea, A flying inverted pendulum, in: 2011 IEEE International Conference on Robotics and Automation, IEEE, 2011, pp. 763-770.

[15] F. Augugliaro, A. P. Schoellig, R. D'Andrea, Dance of the flying machines: Methods for designing and executing an aerial dance choreography, IEEE Robotics \& Automation Magazine 20 (4) (2013) 96-104.

[16] P. Lottes, R. Khanna, J. Pfeifer, R. Siegwart, C. Stachniss, Uav-based crop and weed classification for smart farming, in: 2017 IEEE International Conference on Robotics and Automation (ICRA), IEEE, 2017, pp. 3024-3031.

[17] P. Tripicchio, M. Satler, G. Dabisias, E. Ruffaldi, C. A. Avizzano, Towards smart farming and sustainable agriculture with drones, in: 2015 International Conference on Intelligent Environments, IEEE, 2015, pp. $140-143$.

[18] J. Gu, T. Su, Q. Wang, X. Du, M. Guizani, Multiple moving targets surveillance based on a cooperative network for multi-uav, IEEE Communications Magazine 56 (4) (2018) 82-89.

[19] E. Semsch, M. Jakob, D. Pavlicek, M. Pechoucek, Autonomous uav surveillance in complex urban environments, in: 2009 IEEE/WIC/ACM International Joint Conference on Web Intelligence and Intelligent Agent Technology, Vol. 2, IEEE, 2009, pp. 82-85.

[20] S. Jung, Y. Jo, Y.-J. Kim, Flight time estimation for continuous surveillance missions using a multirotor uav, Energies 12 (5) (2019) 867.

[21] S. Cho, V. Mishra, Q. Tao, P. Vamell, M. King-Smith, A. Muni, W. Smallwood, F. Zhang, Autopilot design for a class of miniature autonomous blimps, in: 2017 IEEE Conference on Control Technology and Applications (CCTA), IEEE, 2017, pp. 841-846.

[22] M. A. Boon, A. P. Drijfhout, S. Tesfamichael, Comparison of a fixed-wing and multi-rotor uav for environmental mapping applications: a case study, ISPRS - International Archives of the Photogrammetry, Remote Sensing and Spatial Information Sciences (2017) 47-54.

[23] A. Vuruskan, B. Yuksek, U. Ozdemir, A. Yukselen, G. Inalhan, Dynamic modeling of a fixed-wing vtol uav, in: 2014 international conference on unmanned aircraft systems (ICUAS), IEEE, 2014, pp. 483-491.

[24] S. H. Song, H. W. Shon, G. Y. Yeon, H. R. Choi, Design and implementation of cloud-like soft drone s-cloud, in: 2018 IEEE/RSJ International Conference on Intelligent Robots and Systems (IROS), IEEE, 2018, pp. 1-9.

[25] S. H. Song, G. Y. Yeon, H. W. Shon, H. R. Choi, Design and control of soft unmanned aerial vehicle "s-cloud", IEEE/ASME Transactions on Mechatronics 26 (1) (2020) 267-275.

[26] M. C. Achtelik, J. Stumpf, D. Gurdan, K.-M. Doth, Design of a flexible high performance quadcopter platform breaking the mav endurance record with laser power beaming, in: 2011 IEEE/RSJ International Conference on Intelligent Robots and Systems, IEEE, 2011, pp. 5166-5172.

[27] K. P. Jain, M. W. Mueller, Flying batteries: In-flight battery switching to increase multirotor flight time, in: 2020 IEEE International Conference on Robotics and Automation (ICRA), IEEE, 2020, pp. 3510-3516.

[28] C. Wan, N. Kingry, R. Dai, Design and autonomous control of a solar-power blimp, in: 2018 AIAA Guidance, Navigation, and Control Conference, 2018, p. 1588.

[29] P. González, W. Burgard, R. Sanz Domínguez, J. López Fernández, Developing a low-cost autonomous indoor blimp, Journal of Physical Agents 3 (1) (2009) 43-52.

[30] B. Lonneville, C. Stal, B. De Roo, B. De Wit, A. De Wulf, P. De Maeyer, Helium balloons for 3d modelling: off to a flying start?, in: Low Cost 3D, Papers of the workshop, 2014.

[31] D. Ikeda, Blade-free drone, NTT DOCOMO Technical Journal 21 (4) (2020) 37-43.

[32] E. F. Frank, Nittany grotto news 32 (5) (1985) 10. 